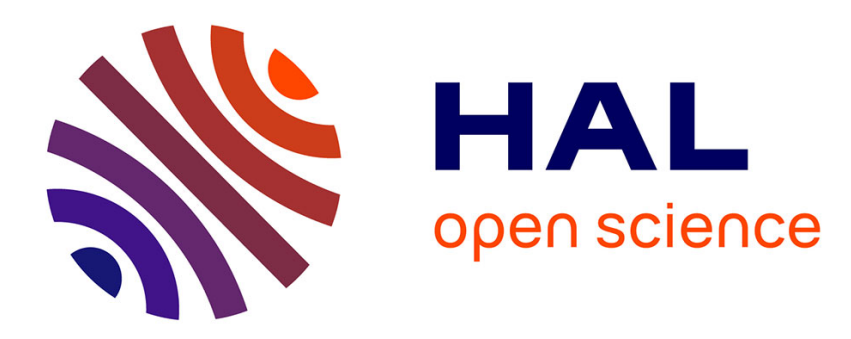

\title{
Ensemble d'acquisition et de traitement en temps réel
}

F. Merchez, M. Tournier

\section{To cite this version:}

F. Merchez, M. Tournier. Ensemble d'acquisition et de traitement en temps réel. Revue de Physique Appliquée, 1969, 4 (2), pp.139-140. 10.1051/rphysap:0196900402013900 . jpa-00243183

\section{HAL Id: jpa-00243183 https://hal.science/jpa-00243183}

Submitted on 1 Jan 1969

HAL is a multi-disciplinary open access archive for the deposit and dissemination of scientific research documents, whether they are published or not. The documents may come from teaching and research institutions in France or abroad, or from public or private research centers.
L'archive ouverte pluridisciplinaire HAL, est destinée au dépôt et à la diffusion de documents scientifiques de niveau recherche, publiés ou non, émanant des établissements d'enseignement et de recherche français ou étrangers, des laboratoires publics ou privés. 


\title{
ENSEMBLE D'ACQUISITION ET DE TRAITEMENT EN TEMPS RÉEL
}

\author{
F. MERGHEZ et M. TOURNIER, \\ Labsratoire de Physique Nucléaire, Institut des Sciences Nucléaires de Grenoble.
}

\begin{abstract}
Résumé. - Les résultats de l'expérience ${ }^{6} \mathrm{Li}(\mathrm{n}, \mathrm{p})$ sont analysés en temps réel par un ensemble d'acquisition et de traitement de données. Réalisé au laboratoire à partir d'éléments standard, cet ensemble, d'une grande souplesse d'utilisation, est particulièrement intéressant pour de faibles taux d'acquisition.
\end{abstract}

Abstract. - The results of the ${ }^{6} \mathrm{Li}(\mathrm{n}, \mathrm{p})$ experiment are analyzed in real time by a data acquisition and processing system. Constructed in the laboratory with standard devices this system, characterized by a great flexibility of utilisation is specially interesting for low acquisition rates.

I. Introduction. - L'ensemble d'acquisition et de traitement de données présenté ici permet, en temps réel, le contrôle constant de la stabilité générale de l'électronique analogique, le stockage des résultats bruts ainsi que les différents traitements nécessaires à l'exploitation théorique des résultats. Get ensemble est pour l'instant connecté à l'expérience ${ }^{6} \mathrm{Li}(\mathrm{n}, \mathrm{p})^{6} \mathrm{He}$ dont les résultats complètent une étude plus générale [1], [2] des transitions $\left({ }^{6} \mathrm{Li}, T=0\right)$ vers le triplet isobarique $(A=6, T=1)$.

II. Dispositif expérimental. - Le dispositif expérimental ( fig. 1) ainsi que les difficultés propres à ce genre d'expérience ont été publiés [3]. Nous employons

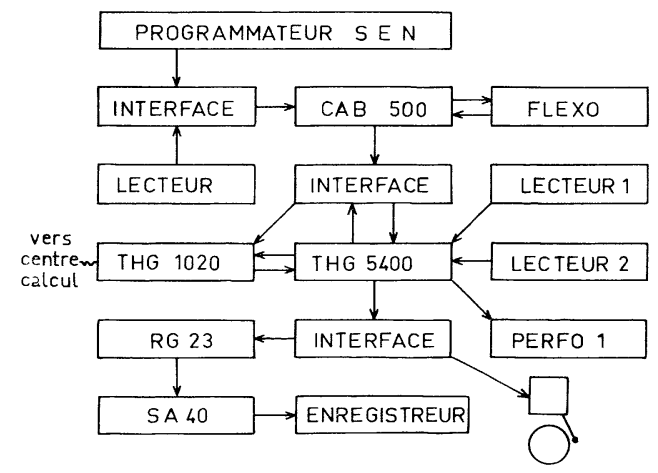

FIG. 1. - Schéma logique du dispositif expérimental.

maintenant un télescope à trois détecteurs en coïncidence quadruple (20 ns) avec le photomultiplicateur qui détecte les alpha associés aux neutrons. Le signal de chaque détecteur est converti en numérique, les deux ou trois nombres obtenus $\left(\Delta E_{1}, \Delta E_{2}, E\right)$ ainsi que le temps et le nombre d'alpha sont alors transmis au programmateur SEN. Le conditionneur d'enre- gistrement signale au calculateur CAB 500 qu'il est prêt à envoyer les données. Les informations sont envoyées " en série » sur l'entrée du lecteur optique. Après traitement dans le calculateur, plusieurs possibilités sont offertes : a) envoyer au centre de calcul, par l'intermédiaire d'une ligne téléphonique, deux modulateurs-démodulateurs, un PDP 8 , à l'ordinateur IBM 7044, les événements simplement triés. Les résultats des calculs peuvent être reçus simultanement par l'intermédiaire de la THG 5400 et aiguillés par programme sur les périphériques souhaités [4]. Cette solution a dû être abandonnée; $b$ ) stocker sur bande perforée les événements après tri et condensation; c) imprimer les résultats et certaines indications de fonctionnement; $d$ ) utiliser la sortie «Perforateur 2 » de la THG 5400, ce qui permet de tracer directement les spectres. Un code spécial envoyé sur cette sortie commande le passage de l'analyseur SA 40 Intertechnique employé en multiéchelles sur stockage et les contenus des canaux sont envoyés sous forme de deux chiffres par l'intermédiaire du RG 23. Le spectre complet étant transmis, l'analyseur repasse soit en lecture, soit en impression. Le spectre est alors visible ou tracé sur l'enregistreur IER. Un autre code permet de déclencher une alarme en cas d'anomalie constatée par la CAB.

III. Exploitation des résultats. - Chaque événement lu est d'abord testé ( fig. 2). Seuls les événements dont les paramètres sont compatibles avec les conditions expérimentales sont retenus, condensés puis stockés sur bande perforée par blocs de 10 événements. Une transformation linéaire permet ensuite de calculer l'énergie perdue par la particule dans chaque détecteur. Selon que cette particule traverse deux ou trois détecteurs, on peut la définir par sa perte d'énergie $\Delta E=\left(\Delta E_{1}+\Delta E_{2}\right.$ ou $\left.\Delta E_{1}\right)$ et son énergie totale $E_{\mathrm{T}} ;$ 


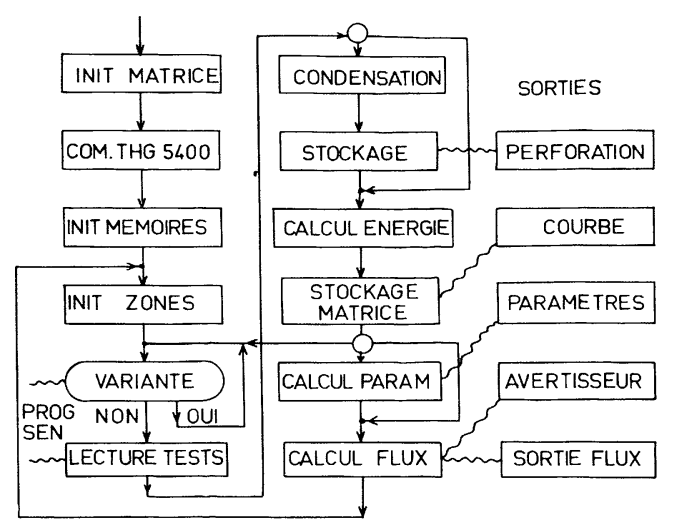

FIG. 2. - Organigramme du programme de traitement.

suivant les cas, l'événement est donc rangé dans l'une des deux matrices $\left(\Delta E, E_{\mathrm{T}}\right)$.

On calcule ensuite les caractéristiques $\left(\overline{\Delta E}, \overline{E_{\mathrm{T}}}\right.$, $\sigma(\Delta E)$ et $\sigma(E))$ de deux zones préalablement définies pour chaque matrice, l'invariance de ces quantités, imprimées pour des séquences prédéterminées, indiquant en continu la stabilité et les performances de l'ensemble de détection. Enfin, d'autres indications dont le choix n'est pas limitatif sont aussi calculées et imprimées à intervalles réguliers (flux partiels, flux intégrés, flux relatifs dans les détecteurs, etc.), toute variation importante de l'une de ces valeurs pouvant entraîner une action extérieure immédiate.

A la fin de chaque expérience, l'impression des matrices et des divers paramètres correspondants donne toutes les informations nécessaires (identité, énergie et nombre de particules provenant d'une réaction donnée). Le spectre en énergie correspondant à chaque genre de particule peut être alors visualisé et imprimé sur un traceur de courbe, cette opération pouvant se faire automatiquement à la fin de séquences prédéterminées.

D'autres programmes, enfin, stockés en permanence dans la $\mathrm{CAB}$ permettent la résolution de problèmes propres à l'expérience en cours ainsi que le traitement ultérieur d'expériences identiques.

IV. Conclusion. - Le calculateur employé ainsi que ses périphériques limitent l'emploi d'un tel ensemble à des expériences où le taux d'événements est faible - de l'ordre d'un événement à la seconde la capacité en mémoires permettant par contre de gérer pour chacun de ces événements de nombreux paramètres, et donc de s'assurer de la stabilité des facteurs expérimentaux.

\section{BIBLIOGRAPHIE}

[1] Perrin (G.), Thèse de $3^{\mathrm{e}}$ cycle, "Contribution à l'étude inélastique ${ }^{6} \mathrm{Li}\left(\mathrm{n}, \mathrm{n}^{\prime}\right)$ à $14 \mathrm{MeV}$ pour le niveau $0^{+}$de $3,56 \mathrm{MeV}$ ).

[2] Merchez (F.), Bouchez (R.), Hoffsweli (R. A.) et Yavin (A. I.), J. Physique, 1968, 29, 969.
[3] Merchez (F.), Helleboid (J. M.), Pouxe (J.), PerRIN (G.) et BENEDETTI (C.), LYCEN, 67-38, 1967, 201.

[4] OudIN (J.), Automatisme, 1968, 9. 